\title{
G359.97-0.038: A Hard X-Ray Filament Associated with a Supernova Shell-Molecular
} Cloud Interaction

Nynka, Melania; Hailey, Charles J.; Zhang, Shuo; Morris, Mark M.; Zhao, Jun-Hui; Goss, Miller; Bauer, Franz E.; Boggs, Stephen E.; Craig, William W.; Christensen, Finn Erland

Total number of authors:

11

Published in:

Astrophysical Journal

Link to article, DOI:

$10.1088 / 0004-637 X / 800 / 2 / 119$

Publication date:

2015

Document Version

Publisher's PDF, also known as Version of record

Link back to DTU Orbit

Citation (APA):

Nynka, M., Hailey, C. J., Zhang, S., Morris, M. M., Zhao, J-H., Goss, M., Bauer, F. E., Boggs, S. E., Craig, W. W., Christensen, F. E., \& Gotthelf, E. V. (2015). G359.97-0.038: A Hard X-Ray Filament Associated with a Supernova Shell-Molecular Cloud Interaction. Astrophysical Journal, 800(2), [119]. https://doi.org/10.1088/0004$637 X / 800 / 2 / 119$

\section{General rights}

Copyright and moral rights for the publications made accessible in the public portal are retained by the authors and/or other copyright owners and it is a condition of accessing publications that users recognise and abide by the legal requirements associated with these rights.

- Users may download and print one copy of any publication from the public portal for the purpose of private study or research.

- You may not further distribute the material or use it for any profit-making activity or commercial gain

- You may freely distribute the URL identifying the publication in the public portal 


\title{
G359.97-0.038: A HARD X-RAY FILAMENT ASSOCIATED WITH A SUPERNOVA SHELL-MOLECULAR CLOUD INTERACTION
}

\author{
Melania Nynka $^{1}$, Charles J. Hailey ${ }^{1}$, Shuo Zhang ${ }^{1}$, Mark M. Morris ${ }^{2}$, Jun-Hui Zhao ${ }^{3}$, Miller Goss $^{4}$, \\ Franz E. Bauer ${ }^{5,6,7}$, Stephen E. Boggs ${ }^{8}$, William W. Craig ${ }^{8,9}$, Finn E. Christensen ${ }^{10}$, Eric V. Gotthelf ${ }^{1}$, \\ Fiona A. Harrison ${ }^{11}$, Kaya Mori ${ }^{1}$, Kerstin M. Perez ${ }^{1}$, Daniel Stern ${ }^{12}$, and William W. Zhang ${ }^{13}$ \\ ${ }^{1}$ Columbia Astrophysics Laboratory, Columbia University, New York, NY 10027, USA \\ ${ }^{2}$ Department of Physics and Astronomy, University of California, Los Angeles, CA 90095, USA \\ ${ }^{3}$ Harvard-Smithsonian Center for Astrophysics, 60 Garden Street, MS 78, Cambridge, MA 02138, USA \\ ${ }^{4}$ NRAO, P.O. Box O, Socorro, NM 87801, USA \\ ${ }^{5}$ Instituto de Astrofísica, Facultad de Física, Pontificia Universidad Católica de Chile, 306, Santiago 22, Chile \\ ${ }^{6}$ Millennium Institute of Astrophysics, Santiago, Chile \\ ${ }^{7}$ Space Science Institute, 4750 Walnut Street, Suite 205, Boulder, CO 80301, USA \\ ${ }^{8}$ Space Sciences Laboratory, University of California, Berkeley, CA 94720, USA \\ ${ }^{9}$ Lawrence Livermore National Laboratory, Livermore, CA 94550, USA \\ ${ }^{10}$ DTU Space-National Space Institute, Technical University of Denmark, Elektrovej 327, DK-2800 Lyngby, Denmark \\ ${ }^{11}$ Cahill Center for Astronomy and Astrophysics, California Institute of Technology, Pasadena, CA 91125, USA \\ 12 Jet Propulsion Laboratory, California Institute of Technology, Pasadena, CA 91109, USA \\ ${ }^{13}$ NASA Goddard Space Flight Center, Greenbelt, MD 20771, USA \\ Received 2014 September 29; accepted 2014 December 9; published 2015 February 18
}

\begin{abstract}
We present the first high-energy X-ray ( $>10 \mathrm{keV}$ ) observations of the non-thermal filament G359.97-0.038 using the Nuclear Spectroscopic Telescope Array (NuSTAR). This filament is one of approximately $20 \mathrm{X}$-ray filaments of unknown origin located in the central $20 \mathrm{pc}$ region in the Galactic Center near Sgr A*. Its NuSTAR and Chandra broadband spectrum is characterized by a single power law with $\Gamma=1.3 \pm 0.3$ that extends from 2 to $50 \mathrm{keV}$, with an unabsorbed luminosity of $1.3 \times 10^{33} \mathrm{erg} \mathrm{s}^{-1}(d / 8 \mathrm{kpc})^{2}$ in the $2-8 \mathrm{keV}$ band. Despite possessing a cometary $\mathrm{X}$-ray morphology that is typical of a pulsar wind nebula (PWN) in high-resolution Chandra imaging, our spatially resolved Chandra spectral analysis found no significant spectral softening along the filament as would be expected from particle synchrotron cooling. Coincident radio emission is detected using the Very Large Array at 5.5 and $8.3 \mathrm{GHz}$. We examine and subsequently discard a PWN or magnetic flux tube as the origin of G359.97-0.038. We use broadband spectral characteristics and a morphological analysis to show that G359.97-0.038 is likely an interaction site between the shell of Sgr A East and an adjacent molecular cloud. This is supported by CS molecular line spectroscopy and the presence of an $\mathrm{OH}$ maser.
\end{abstract}

Key word: Galaxy: center

\section{INTRODUCTION}

The Galactic Center (GC) is a densely populated region that is home to a number of energetic thermal and non-thermal objects. In addition to Sagittarius $A^{*}$, several supernova remnants (SNRs), H II regions, molecular clouds (MCs), and pulsar wind nebulae (PWNe) have been identified and studied (e.g., Baganoff et al. 2003; Goldwurm 2011). Notable are the many non-thermal filaments (NTFs) observed throughout the GC.

Dust in the Galactic plane obscures the GC at optical and UV wavelengths, requiring observatories operating in the radio, IR, X-ray, and $\gamma$-ray bands to reveal the complex structure. As such, large-scale NTFs were first observed in the radio band. Extending up to tens of parsecs in length yet with widths of only a few parsecs (e.g., Yusef-Zadeh et al. 1984), NTFs are well-described by a non-thermal power law in the radio band with spectral index $\alpha \sim 0.3\left(S_{v} \propto \nu^{\alpha}\right)$ (Inoue et al. 1984; Reich et al. 2000).

The advent of high-resolution imaging from X-ray observatories such as Chandra and XMM-Newton revealed X-ray emission from smaller NTFs that populate the GC. Three extensive X-ray studies have cataloged over 20 filaments below $10 \mathrm{keV}$ (Muno et al. 2008; Lu et al. 2008; Johnson et al. 2009) while dedicated observations have probed in greater detail several of the brighter filaments. Unlike the large-scale NTFs, these filaments are smaller $(0.1-2 \mathrm{pc})$ in length and are randomly oriented with respect to the Galactic plane (Muno et al. 2008). A few filaments show a tight spatial correlation between their radio and $\mathrm{X}$-ray emission, suggesting in those cases that the same emission mechanism (e.g., synchrotron radiation) is dominant. (e.g., Sgr A-East; Lu et al. 2003; Yusef-Zadeh et al. 2005; Zhang et al. 2014).

A commonly accepted scenario for the small NTFs is a PWN that has been confined to a filamentary structure by either nearby dense material or ram pressure from a fast-moving pulsar, as proposed by Muno et al. (2008). Both can result in an elongated structure with a concentrated region of bright emission at the location of the pulsar (Kargaltsev \& Pavlov 2008). A lack of iron line emission, non-thermal spectra with photon indices $\Gamma \sim 1-3$, and luminosities from $\sim 10^{32}$ to $10^{33} \mathrm{erg} \mathrm{s}^{-1}(2-8 \mathrm{keV})$ are all characteristics of PWNe (Muno et al. 2008). G359.95-0.04 is one such PWN filament. Located $\sim 9^{\prime \prime}$ from SgrA*, it has been identified as a PWN due to its morphological properties and the observed spectral steepening, indicative of synchrotron burnoff in the nebula (Wang et al. 2006). While no pulsations or variability in either the radio or X-ray bands have been detected from these filaments, this is possibly due to the large dispersion measure of the GC.

We discuss here the filament G359.97-0.038, located $\sim 45^{\prime \prime}$ northeast of Sgr A*. Of the approximately 20 filaments in the 
GC observed by Chandra and XMM-Newton, G359.97-0.038 is the second to be detected in the hard X-ray band by Nuclear Spectroscopic Telescope Array (NuSTAR). Multi-wavelength analysis, both spectral and spatial, has already shown Sgr AEast (Sgr A-E) to likely be a magnetic flux tube and not a PWN (Zhang et al. 2014). Similarly for G359.97-0.038, examining NuSTAR data concurrently with radio and soft X-ray observations provides broadband information that can elucidate its nature.

G359.97-0.038 has been previously observed in the X-ray band below $10 \mathrm{keV}$ (Muno et al. 2008; Lu et al. 2008; Johnson et al. 2009). Muno et al. (2008) measured a power-law spectrum with a photon index of $\Gamma=1.7_{-0.3}^{+0.5}$ and a $2-8 \mathrm{keV}$ luminosity of $1 \times 10^{33} \mathrm{erg} \mathrm{s}^{-1}$. Lu et al. (2008) and later Johnson et al. (2009) were able to increase the exposure and reported photon indices of $\Gamma=1.4_{-0.5}^{+0.9}$ and $\Gamma=1.4_{-0.3}^{+0.8}$, respectively. Assuming a distance of $8 \mathrm{kpc}$, the $2-8 \mathrm{keV}$ luminosities were $L_{\mathrm{X}}=$ $8.4 \times 10^{32} \mathrm{erg} \mathrm{s}^{-1}$ and $L_{\mathrm{X}}=1.5 \times 10^{33} \mathrm{erg} \mathrm{s}^{-1}$. No pulsations were detected.

Based on its spectral and spatial properties, G359.97-0.038 was first tentatively classified as a PWN by Muno et al. (2008). Lu et al. (2008) attempted to confirm this hypothesis by analyzing the filament in two segments to search for the spectral softening that would be expected from a PWN. The first region encloses the point-like object located toward the SW of the filament and the second region captured the streaming tail to the NE. However, these results proved inconclusive. The photon indices of the tail and the head, $\Gamma=1.2_{-0.9}^{+1.1}$ and $\Gamma=1.6_{-0.9}^{+0.4}$, respectively, agree within uncertainties and these observations were not able to confirm G359.97-0.038 as a PWN.

In this work we present Chandra and NuSTAR observations to further explore the filament. NuSTAR extends the X-ray spectrum up to $50 \mathrm{keV}$ for the first time, while the Chandra data improve the low-energy exposure from that previously reported, allowing for improved spatially resolved spectroscopy to probe spectral softening along the filament. Radio data at 5.5 and $8.3 \mathrm{GHz}$ provide spectral and morphological information at lower energies. We also consider $\gamma$-ray data from the Fermi observatory. We utilize the resulting spectral energy density (SED) analysis from the radio to $\gamma$-ray bands and compare the emission from G359.97-0.038 to that expected from a typical PWN.

\section{OBSERVATIONS AND DATA REDUCTIONS}

NUSTAR, the first focusing telescope in orbit operating in the 3-79 keV energy band, provides a unique perspective of the GC. Two co-aligned optics pairs with corresponding focal planes referred to as focal plane modules (FPMA and FPMB), have angular resolution of $18^{\prime \prime}$ full width at half-maximum (FWHM), $58^{\prime \prime}$ half power diameter (HPD), an energy resolution of $400 \mathrm{eV}$ $(\mathrm{FWHM})$ at $10 \mathrm{keV}$, and a $12^{\prime} \times 12^{\prime}$ field of view (Harrison et al. 2013).

NUSTAR data were obtained from three Sgr A* observations spanning 2012 July 20 to 2012 October 10 (Barrière et al. 2014). Other NUSTAR observations were also centered on Sgr A*; however, those data were not useful since the emission from G359.97-0.038 was obscured by either bright X-ray transients or the magnetar SGR J1745-29 (Mori et al. 2013).

Each individual observation, listed in Table 1, was registered to J2000 coordinates using known Chandra positions of objects detected in the NuSTAR field of view following the procedure described in Nynka et al. (2013). The centroiding errors (90\%
Table 1

NUSTAR Log of G359.97-0.038 Observations

\begin{tabular}{lccc}
\hline \hline \multicolumn{4}{c}{$N u S T A R$ ObsIDs } \\
\hline ObsID & $\begin{array}{c}\text { Start Date } \\
(\text { UTC })\end{array}$ & $\begin{array}{c}\text { Exposure } \\
(\mathrm{ks})\end{array}$ & Target \\
\hline 30001002001 & 2012 Jul 20 & 166.2 & Sgr A* \\
30001002003 & 2012 Aug 4 & 83.8 & Sgr A* \\
30001002004 & 2012 Oct 16 & 53.6 & Sgr A* \\
\hline & Chandra ACIS-I ObsIDs & \\
\hline
\end{tabular}

1561b, 2943, 2951, 2952, 2953, 2954, 3392, 3393, 3549

$3663,3665,4684,5360,5950,59515952,5953,5954$ $6363,6639,6640,6642,6643,6644,6645,6646,7554$ $7556,7557,7558,9169,9170,9171,9172,9173,9174$ $10556,11843,12949,13016,13017,13438,13508$

Notes. The exposure times listed are corrected for good time intervals. The total Chandra exposure time is $1.47 \times 10^{6} \mathrm{~s}$, spanning $11 \mathrm{yr}$ (2000-2011) of observations.

confidence level) are $\sim 3^{\prime \prime}$ in both right ascension and declination, which combine for a total position uncertainty of $3^{\prime \prime}$.

We used nupipeline (NuSTARDAS v. 1.1.1) to process all NuSTAR observations and generate response files and exposure maps for spectral and spatial analysis. XSPEC v. 12.8.1 from HEASOFT v. 6.14 was used to determine spectral parameters. Photon arrival times were corrected for onboard clock drift and precessed to the solar system barycenter using the JPL-DE200 ephemeris and the Chandra position. The nominal NuSTAR timing absolute accuracy is $3 \mathrm{~ms}$.

We utilize the available Chandra Advanced CCD Imaging Spectrometer (ACIS-I) observations that span $11 \mathrm{yr}$, for a cumulative exposure of $1.47 \mathrm{Ms}$ (see Table 1). This is an increase of $\sim 60 \%$ from the $927 \mathrm{ks}$ analyzed by Muno et al. (2008), and an increase of $\sim 30 \%$ in the number of photons presented by Johnson et al. (2009). The data were extracted following the point source and diffuse emission recipes from the ACIS EXTRACT (AE) software package (Broos et al. 2010), along with CIAO version 4.5 and associated CALDB version 4.5.6. AE automates the process of extracting events from individual ObsIDs, generating spectral products, and ultimately combining these spectral products into a composite data set for analysis. As input for AE, we generated cleaned, flare-free event lists and byproducts following standard CIAO analysis threads. For point sources, AE generates point-spread function (PSF) corrections to compensate for the varying PSF size as a function of energy. Given that the encircled energy fraction for even a $10^{\prime \prime}$ diameter circular region can be as low as $\sim 92 \%$ at $8 \mathrm{keV}$, and that the regions presented here were $7^{\prime \prime}$ or narrower in width, we applied a PSF correction to the extracted spectra. These differential corrections amount to $\sim 1 \%$ and $\sim 10 \%$ at $1.4 \mathrm{keV}$ and $8 \mathrm{keV}$, respectively.

The detailed procedures for the reduction of the Very Large Array data are described in Zhao et al. (2009) and Zhao et al. (2013). Tapering and weighting the data, Sgr A East and the surrounding region were imaged at 5.5 and $8.3 \mathrm{GHz}$ with a common clean beam 1".65 × 1".65 (10.3) in size. The flux densities were determined by integrating the emission from the extracted regions. A mean brightness of 8 and $4 \mu \mathrm{Jy}_{\text {pixel }}{ }^{-1}$ in the surrounding area of G359.97-0.038 was estimated for the 5.5 and $8.3 \mathrm{GHz}$ images, respectively. Using the values of the mean brightness, the raw flux densities were corrected for this background emission. 


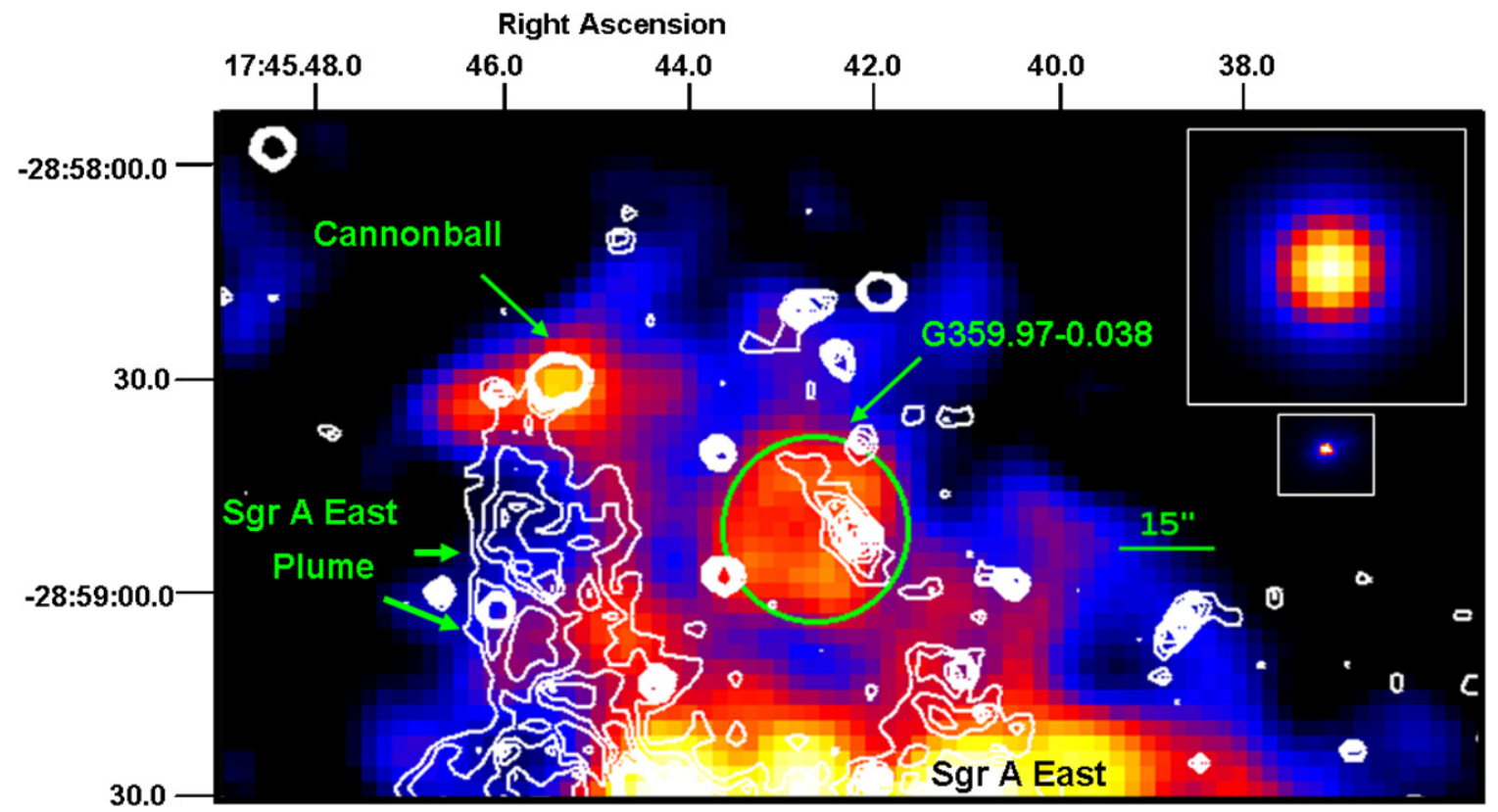

Figure 1. NUSTAR 10-40 keV mosaicked and exposure map-corrected image. A Gaussian kernel with FWHM $=3$ pixels $\left(\sim 12^{\prime \prime}\right)$ was applied to the mosaicked image. The linear scaling color range was chosen to highlight the emission from G359.97-0.038. Chandra $2-8 \mathrm{keV}$ contours overlaid in white. The green circle with $15^{\prime \prime}$ radius indicates the NUSTAR data extraction region. The top and bottom inserts indicate the NUSTAR and Chandra ACIS-I on-axis PSFs, respectively.

\section{IMAGE ANALYSIS}

We created mosaicked, exposure-corrected images from the NuSTAR observations listed in Table 1 in various energy bands. The $10-40 \mathrm{keV}$ image is shown in Figure 1. This energy range was chosen to reduce the contribution from the thermal emission of Sgr A East that dominates the GC below $10 \mathrm{keV}$ due to the broad NUSTAR PSF. Visible in the top left of the image is the Cannonball, a high-velocity neutron star with hard X-ray emission (Nynka et al. 2013). G359.97-0.038 is located in the middle of the image, indicated by the green circle. Overlaid in white are the $2-8 \mathrm{keV}$ contours from Chandra.

G359.97-0.038 is detected by NUSTAR above $20 \mathrm{keV}$ with a significance of $\sim 5 \sigma$. We investigated the high-energy morphology of G359.97-0.038 by following the procedure outlined in Nynka et al. (2013). Briefly, this involves fitting a symmetric two-dimensional Gaussian profile convolved with the NUSTAR PSF by using the CIAO fitting application Sherpa (Fruscione et al. 2006). The centroid position matched within the uncertainty to the location of the X-ray peak emission determined by Chandra.

A high-resolution $5.5 \mathrm{GHz}$ image is presented in Figure 2 with the Chandra 2-8 keV contours of G359.97-0.038 in white. The central bright point-like object in the X-ray band is coincident with a region of bright $5.5 \mathrm{GHz}$ emission. There are several important differences to note, however. The radio emission is more compact than the X-ray emission, with a length of $\sim 10^{\prime \prime}$ compared to $\sim 20^{\prime \prime}$ observed by Chandra. Additionally, the filamentary structure from $2-8 \mathrm{keV}$ extends NE from the point-like object while the radio emission extends SE.

\section{SPECTRAL ANALYSIS}

\subsection{NuSTAR Hard Energy X-Ray Spectrum}

We extracted NUSTAR data from the observations listed in Table 1. The circular region used for source extraction $\left(r=15^{\prime \prime}\right)$ was centered on the bright X-ray emission in the NUSTAR image

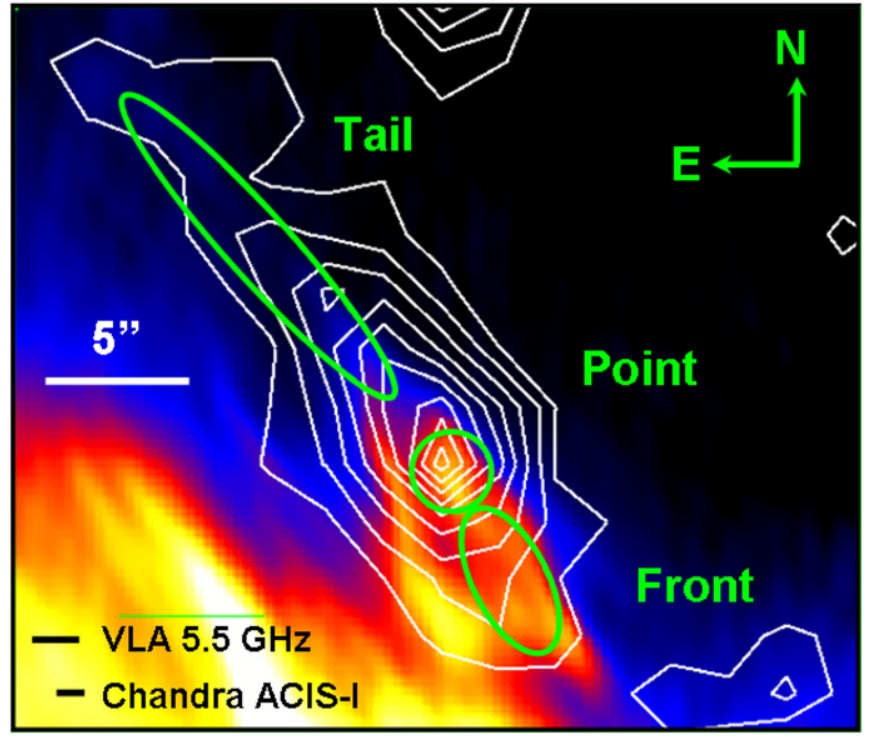

Figure 2. 5.5 GHz VLA image from Zhao et al. (2013) centered on G359.970.038 . The same Chandra contours from Figure 1 are overlaid in white. Three green regions, "Tail," "Point," and "Front," indicate the extraction regions for $5.5 \mathrm{GHz}$ and $8.31 \mathrm{GHz}$ data. For reference, the resolutions of the VLA and Chandra ACIS-I instruments are indicated in black in the bottom right.

(R.A. $17^{\mathrm{h}} 45^{\mathrm{m}} 42^{\mathrm{s}} \cdot 1$, decl. $-28^{\circ} 58^{\prime} 43^{\prime \prime} 48$, J2000). This region size was chosen to reduce the contamination of thermal emission from nearby Sgr A East. The spectrum of G359.97-0.038 was modeled as an absorbed power law using tbabs for the hydrogen column with the molecular abundances and cross sections set to wilms and vern, respectively (Wilms et al. 2000; Verner et al. 1996).

The NUSTAR data are subject to four different background components: detector background, cosmic X-ray background (CXB), thermal emission from Sgr A East, and hard X-ray 


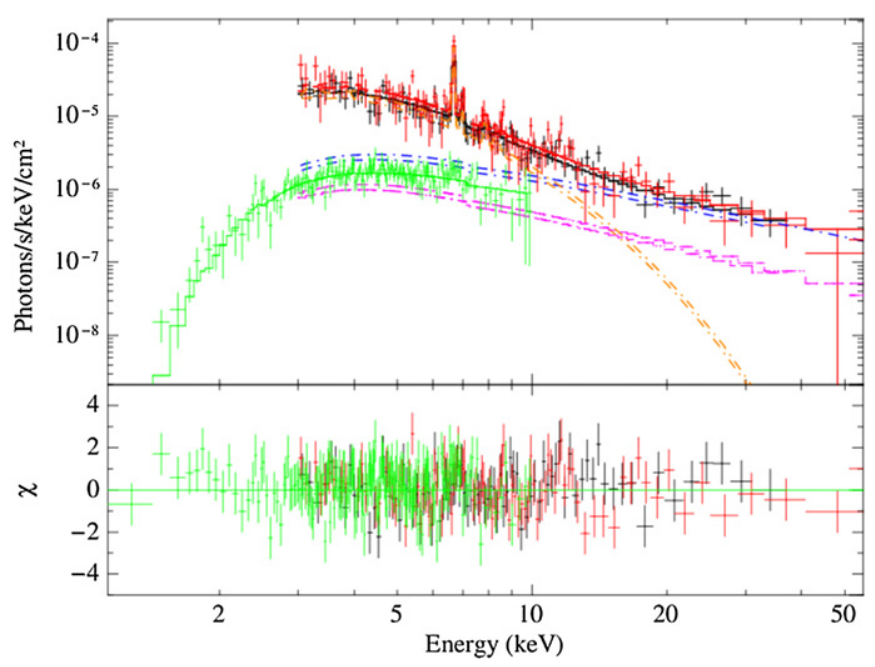

Figure 3. NUSTAR and Chandra unfolded joint fit spectra. The black and red data points correspond to NuSTAR FPMA and FPMB data, respectively, while Chandra data are represented by green. The components of the best-fit spectral model are colored orange (Sgr A East), pink (high-energy Galactic diffuse emission), and blue (G359.97-0.038).

diffuse emission. We subtracted the detector and CXB emission and modeled the latter two, each outlined below in further detail.

First, spectral subtraction was used to remove detector background and CXB. This includes emission intrinsic to the detector chip itself as well as emission that bypasses the optics and falls directly on the detectors to form a non-uniform pattern (Wik et al. 2014). This pattern is the same for observations that have similar position angles, as do those listed on Table 1. We can therefore pair the observations such that the detector region containing G359.97-0.038 in one observation contains only the detector background in another observation. A more detailed explanation can be found in Krivonos et al. (2014).

Next, we modeled the thermal contribution from Sgr A East, the edge of which is located $\sim 20^{\prime \prime}$ from G359.97-0.038. Any extraction region centered on G359.97-0.038 will contain emission from Sgr A East due to the NuSTAR HPD of 58". We therefore included a thermal component apec in the NuSTAR spectral model. The temperature as well as the abundance were left as variable parameters.

Last, we incorporated a model of the diffuse hard X-ray emission (DHXE) that is visible in the GC above $20 \mathrm{keV}$ (Perez et al. 2015). First revealed by $N U S T A R$, this emission is aligned along the Galactic plane and is approximately $8 \mathrm{pc} \times 4 \mathrm{pc}$ in size. It is thought to be caused by unresolved faint point sources. Its spectrum is well modeled by a single non-thermal power law with a photon index of $\Gamma=1.5$. The normalization was chosen to correctly account for the flux contained in the $r=15^{\prime \prime}$ extraction region based on the spatial model presented in Perez et al. (2015).

In order to better constrain the low-energy behavior as well as the absorption column, the NuSTAR data were jointly fit with data from Chandra. The narrow Chandra PSF (FWHM $<1^{\prime \prime}$ ) allows the extracted spectra to contain only G359.97-0.038 emission and detector background, which was subtracted using a region on the same detector chip away from the source. The Chandra data were fit with an absorbed power-law model, with all of the parameters linked to the neutral hydrogen column density and power-law components in the NUSTAR model. A multiplicative parameter const was introduced in order to
Table 2

$N u S T A R+$ Chandra Spectral Analysis Results

\begin{tabular}{lc}
\hline \hline Parameter & Best-fit Values \\
\hline$N_{\mathrm{H}}\left(10^{23} \mathrm{~cm}^{-2}\right)$ & $1.3 \pm 0.2$ \\
Power-law index $\Gamma$ & $1.3_{-0.2}^{+0.3}$ \\
$\chi_{v}^{2}($ dof $)$ & $0.97(363)$ \\
$F_{\mathrm{X}}(2-8 \mathrm{keV})^{\mathrm{a}}$ & $9.8 \times 10^{-14}$ \\
$L_{\mathrm{X}}(2-8 \mathrm{keV})^{\mathrm{b}}$ & $1.3 \times 10^{33}$ \\
$F_{\mathrm{X}}(20-40 \mathrm{keV})^{\mathrm{a}}$ & $3.4 \times 10^{-14}$ \\
$L_{\mathrm{X}}(20-40 \mathrm{keV})^{\mathrm{b}}$ & $2.6 \times 10^{33}$ \\
\hline
\end{tabular}

Notes. Best-fit parameters of G359.97-0.038, from joint fit of NUSTAR and Chandra data. The errors are $90 \%$ confidence level. Flux values are obtained from the filament absorbed power-law component.

${ }^{a}$ Flux units are erg s ${ }^{-1} \mathrm{~cm}^{-2}$.

${ }^{\mathrm{b}}$ Luminosity units are erg $\mathrm{s}^{-1}$.

account for any normalization offsets between the Chandra and NUSTAR data sets.

The NuSTAR data set is modeled by const $*$ tbabs $*\left(\right.$ po $_{\mathrm{G} 359.97}+$ apec + po $\left._{\mathrm{DHXE}}\right)$

while the Chandra spectral model is tbabs $*\left(\right.$ po $\left._{\mathrm{G} 359.97}\right)$.

The resultant spectral fit is shown in Figure 3 and in Table 2. This is the first direct detection of G359.97-0.038 above $10 \mathrm{keV}$, and clearly shows emission up to $40 \mathrm{keV}$. The bestfit photon index for G359.97-0.038 is $\Gamma=1.3_{-0.2}^{+0.3}$, consistent with previously reported values of the whole region ( $\mathrm{Lu}$ et al. 2008; Johnson et al. 2009). The best-fit column density is $N_{\mathrm{H}}=(1.3 \pm 0.2) \times 10^{23} \mathrm{~cm}^{-2}$. The thermal component was best fit to $k T=3.6 \pm 0.5 \mathrm{keV}$, consistent with what is expected near the edge of the Sgr A East SNR shell (Sakano et al. 2004).

As a confirmation of our results, we fit the Chandra data alone with a simple absorbed power-law model. The fit parameters of $N_{\mathrm{H}}=(1.5 \pm 0.2) \times 10^{23} \mathrm{~cm}^{-2}$ and $\Gamma=1.4 \pm 0.3$ are consistent with the results obtained with the joint $N U S T A R$ and Chandra data.

\subsection{Spatially Resolved Spectral Analysis}

We extracted Chandra data from the observations listed in Table 1. We first analyzed the spectrum of the entire filament. Then we analyzed five smaller regions in order to search for spectral softening that would help support a PWN origin of G359.97-0.038. The left of Figure 4 shows the four rectangular subdivisions chosen to study the spectral evolution along the filament, as well as an additional elliptical region labeled "point" centered on the region of brightest emission. The spectra were all fit with an absorbed non-thermal power-law continuum.

The absorption of the subdivisions was fixed to $N_{\mathrm{H}}=$ $1.5 \times 10^{23} \mathrm{~cm}^{-2}$. The results are consistent with the values obtained with joint NUSTAR and Chandra data, described in Section 4.1.

The photon indices shown in Figure 4 indicate a slight spectral softening when moving northeast along the filament, as would be expected for a PWN. However, this trend is not statistically significant as all of the indices overlap within their $90 \%$ uncertainties. 

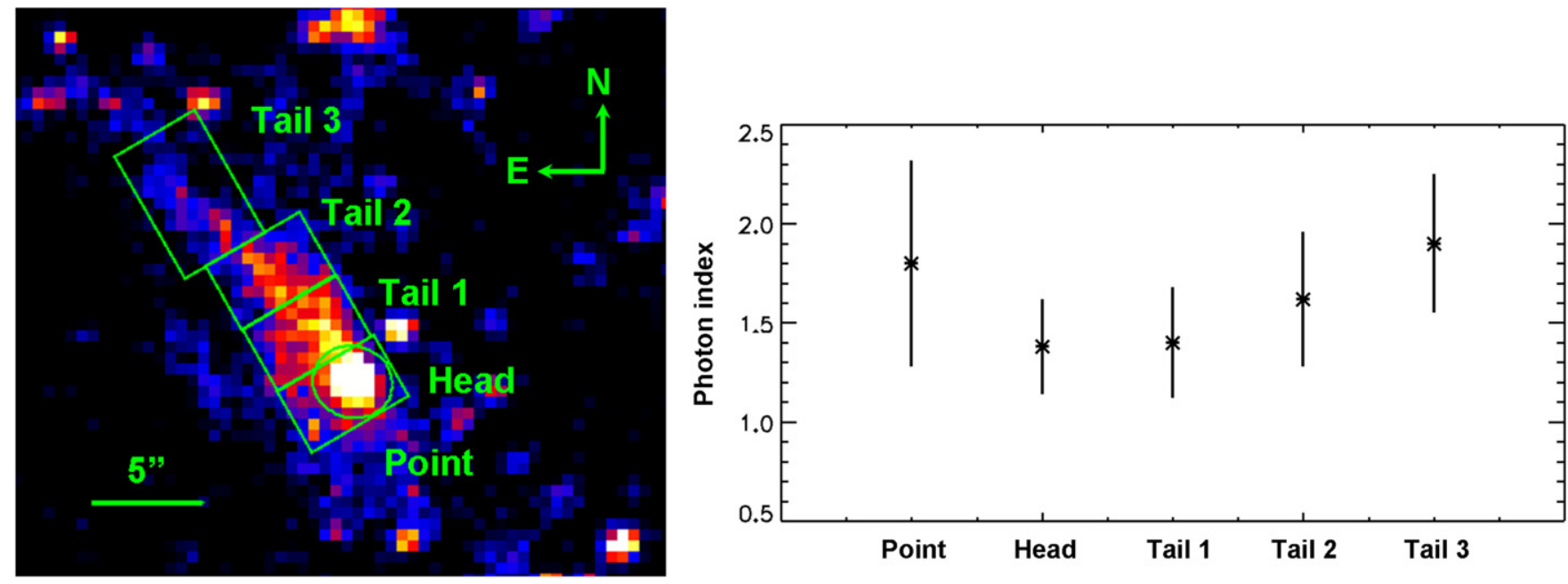

Figure 4. Left: Chandra 0.7-8.0 keV image of G359.97-0.038, covering the same field of view as Figure 2. Overlaid in green are the regions chosen for analysis. "Point" refers to the elliptical region centered on the point-like emission. "Head," "Tail 1, 2, 3" define rectangular extraction regions. The image was smoothed with a Gaussian kernel (FWHM = 1 pixel) and the color is presented in a linear scale. Right: photon indices of G359.97-0.038 obtained with Chandra, with error bars representing $90 \%$ confidence.

Table 3

Radio Spectral Indices

\begin{tabular}{lrrr}
\hline \hline Region & \multicolumn{2}{c}{ Flux Density $(\mathrm{mJy})$} & \multicolumn{1}{c}{ Spectral Index } \\
\cline { 2 - 3 } & \multicolumn{1}{c}{$5.5 \mathrm{GHz}$} & $8.31 \mathrm{GHz}$ & \multicolumn{1}{c}{$\alpha$} \\
\hline Tail & $6.0 \pm 0.3$ & $4.6 \pm 0.3$ & $-0.64 \pm 0.10$ \\
Point & $10.1 \pm 0.3$ & $9.7 \pm 0.4$ & $-0.1 \pm 0.11$ \\
Front & $17.5 \pm 0.4$ & $18.7 \pm 0.4$ & $0.16 \pm 0.06$ \\
\hline
\end{tabular}

Notes. Best-fit radio spectral indices and fluxes from subregions of G359.970.038. The errors are $1 \sigma$ confidence level.

\subsection{Radio Spectral Analysis}

We extracted radio spectra at $5.5 \mathrm{GHz}$ and $8.3 \mathrm{GHz}$ from three regions that coincide with the X-ray regions labeled "Point," "Tail," and "Front" in Figure 2. These regions, 6.8, 24.6, and 12.8 beams in area, respectively, would have spectra with specific correlations to the X-ray spectra if G359.97-0.038 were a PWN, as discussed in Section 5.2. The flux density measurements are dominated by statistical errors, and the spectral results are listed in Table 3.

\section{DISCUSSION}

\subsection{What is G359.97-0.038?}

NuSTAR has detected hard X-ray emission up to $40 \mathrm{keV}$ from the filament G359.97-0.038. After Sgr A-E (Zhang et al. 2014), this is the second detection of an NTF at energies higher than $10 \mathrm{keV}$. Common astrophysical mechanisms that can produce such hard non-thermal X-ray emission include synchrotron radiation, bremsstrahlung, and inverse-Compton (IC) scattering.

The means to distinguish these mechanisms involve the possible astrophysical objects that are generally attributed to NTFs. We therefore examine three possible underlying explanations for G359.97-0.038: a PWN, a magnetic flux tube accelerating local electrons, and a region of interaction between an SNR and an MC. Each has a distinct broadband energy spectrum, thereby allowing us to determine the nature of G359.97-0.038.

Both a PWN and a magnetic flux tube can produce bright synchrotron emission in the X-ray band, generated by charged relativistic electrons interacting with strong magnetic fields. The shape of the resultant PWN SED curve is determined by several parameters such as the age of the nebula, the time-dependent magnetic field, and the spectrum of the relativistic electrons streaming from the pulsar. Multiwavelength SEDs show that synchrotron radiation from a PWN spans from the radio to the hard X-ray band above which the luminosity begins to rapidly decrease. The same relativistic electrons also interact with UV and IR photons from the surroundings to create IC emission bright in $\mathrm{GeV}$ and $\mathrm{TeV} \gamma$-rays. In Section 5.2 we show that G359.97-0.038 cannot be a PWN.

Magnetic flux tubes, or locally enhanced magnetic field lines, interact with nearby relativistic electrons that are accelerated in the magnetic field. While the resultant emission produces synchrotron and IC radiation similar to that of PWNe, differences in magnetic field strengths along with both spectral and morphological characteristics can be used to distinguish a magnetic flux tube from a PWN. In Section 5.3 we argue that G359.97-0.038 is not a magnetic flux tube.

In contrast to both a PWN and flux tube, an SNR-MC interaction will emit X-rays via non-thermal bremsstrahlung radiation. Broadband SED features as well as morphological characteristics of $\mathrm{SN}$ shells known to be interacting with dense MCs match those observed in G359.97-0.038, as presented in Section 5.4 .

\subsection{G359.97-0.038 as a $P W N$}

G359.97-0.038 has been conventionally described as a PWN. A PWN is formed when a relativistic wind of electron/positron pairs streaming from a pulsar experience a termination shock close to the pulsar itself (e.g., Gaensler \& Slane 2006). As the particles propagate outward from the termination shock they emit synchrotron radiation, and the cooling time of the leptons is inversely proportional to their energy $(\tau \propto 1 / E)$. This results in a radially dependent spectrum whose photon index softens at larger distances from the pulsar. Such effects have been wellstudied over a broad X-ray energy range in G21.5-0.9, MSH 15-52, and the Crab (e.g., Nynka et al. 2014; An et al. 2014; K. K. Madsen et al., in preparation). The synchrotron emission for most PWNe is visible from the radio through the X-ray band, while IC emission extends from $\mathrm{GeV}$ to $\mathrm{TeV}$ energies. 
When considering the X-ray morphology from Chandra and NUSTAR alone, G359.97-0.038 indeed resembles a PWN. Visible in the left of Figure 4 are a point-like bright head, which is the presumed location of the pulsar, and an extended tail representing the nebula streaming behind it. Assuming the cometary structure of G359.97-0.038 is caused by the proper motion of the pulsar, the trajectory would be toward the SW along the length of the filament. Several other PWNe exhibit this same elongated morphology, such as the Mouse nebula (Gaensler et al. 2004).

Consistent with a PWN, the non-thermal power-law spectrum shows no evidence of line emission. The power-law photon index of $\Gamma \sim 1.3$ and $2-10 \mathrm{keV}$ luminosity $L_{\mathrm{X}} \sim 1.64 \times$ $10^{33} \mathrm{erg} \mathrm{s}^{-1}$ are also consistent with those of a typical PWN, which have nominal ranges of $\Gamma \sim 1.1-2.4$ and $2-10 \mathrm{keV}$ luminosities from $4 \times 10^{32}$ to $2 \times 10^{35} \mathrm{erg} \mathrm{s}^{-1}$ (e.g., Gaensler \& Slane 2006).

Two other features commonly used to identify PWNe did not provide conclusive evidence as to the nature of G359.97-0.038. Due to the energy-dependent lifetime of synchrotron radiation, PWNe exhibit spectral softening when moving from the pulsar to the edge of the PWN. Soft X-ray analysis of the subdivisions of G359.97-0.038 shows no statistical softening in the spectral indices due to the large uncertainties in the fit parameters (see Figure 4). Second, NuSTAR statistics were too poor to provide a meaningful limit on pulsation from the point-like region, which should contain a pulsar.

However, three key broadband features conclusively rule out the PWN hypothesis for G359.97-0.038. We examine each of these in turn.

First, the radio and X-ray morphologies do not match with those expected from a PWN. The lifetime of synchrotron radiation is energy-dependent, and thus the radio emission of a PWN should have a broader, more diffuse structure than the $\mathrm{X}$-ray emission. The radio emission of a pulsar in motion traces its trajectory. Thus one would expect the peak of the radio emission to trail the bright point-like X-ray source. Figure 2 shows a filamentary structure roughly coincident with the $\mathrm{X}$-ray morphology of G359.97-0.038, as well as a peak in radio emission at the position of the X-ray point-like object. However, the radio image is more compact than the Chandra contours shown in white, and the peak of the $6 \mathrm{~cm}$ emission is located at the putative head of the PWN rather than the tail (assuming the pulsar is indeed moving along a SW direction).

Second, we compare the radio and X-ray spectral indices. The energy-dependent lifetime of synchrotron emission causes a well-defined spectral break $\Delta \equiv \alpha_{x}-\alpha_{r}$ where $\alpha_{x}$ and $\alpha_{r}$ represent the spectral indices in the radio and X-ray bands, respectively. As discussed in Reynolds (2009), the $\Delta=0.5$ nominal value for a PWN is perturbed by the magnetohydrodynamic (MHD) transport mechanisms within a nebula such that observed PWN spectral breaks are greater, increasing to values of $\sim 1$. One such example is the spectral break of $\Delta=0.9$ found in the PWN G21.5-0.9 (Nynka et al. 2014).

With the assumption that G359.97-0.038 is a PWN and its pulsar's proper motion is oriented toward the SW, the nebula would be located in the "tail" regions defined in Figures 4 and 2. Comparing the radio and X-ray spectral parameters within these regions yields an observed break of $\Delta=0.0 \pm 0.3$ for G359.970.038 , which is too low for a PWN.

Last, we compare the broadband emission of G359.970.038 to that expected from a PWN. Extensive one-zone, timedependent MHD models have been well developed that quantify
Table 4

Parameters PWN SED Model

\begin{tabular}{lccc}
\hline \hline Parameter & Symbol & \multicolumn{2}{c}{ Value } \\
\cline { 3 - 4 } & & Case 1 & Case 2 \\
\hline Characteristic age $(\mathrm{kyr})$ & $\tau_{c}$ & 10 & 5 \\
Radius $(\mathrm{pc})$ & $r$ & 0.5 & 2 \\
Magnetic field $(\mu \mathrm{G})$ & $B_{0}$ & 20 & 20 \\
Breaking index & $n$ & 3 & 3 \\
Moment of inertia $\left(\mathrm{g} \mathrm{cm}^{2}\right)$ & $I$ & $10^{45}$ & $10^{45}$ \\
\hline
\end{tabular}

Note. Relevant parameters for SED model of a PWN.

the relationship between the spectral properties of a nebula and parameters such as age, magnetic field strength and spin period. Using a model developed by Zhang et al. (2008), we created SED plots for PWNe with various configurations.

Many model parameters are common for the majority of PWNe and as such were left fixed in this study. The braking index, for example, was fixed at $n=3$, and the moment of inertia was fixed at $10^{45} \mathrm{~g} \mathrm{~cm}^{2}$. Other variables, such as the break energy of the injected electron spectrum, do not have a significant impact on the resultant SED shape.

We first present an SED for a PWN with nominal parameters, shown in the left panel of Figure 5 and detailed in Table 4. This is identified as Case 1. The red overlay represents the NUSTAR spectrum and its uncertainty, while the blue indicates the VLA data. Since it is possible that some or most of the detected radio emission can be produced by the Sgr A East shell itself, the emission presented in Section 4.3 represents an upper limit. Relevant parameters for Case 1 include a magnetic field strength of the nebula of $B_{0}=20 \mu \mathrm{G}$ and a characteristic age of $\tau_{c}=10 \mathrm{kyr}$. The size of the nebula was chosen as $0.5 \mathrm{pc}$ to match the observed length from Chandra. The resultant SED spectrum overpredicts the radio flux while underpredicting the flux in the X-ray band. The model for an older PWN would predict an even lower X-ray flux, and as such we can safely claim that G359.97-0.038 is not a PWN 10 kyr or older.

Next we manipulated the parameters outside of their average ranges to determine whether G359.97-0.038 could be a PWN with rare characteristics. This is presented as Case 2, shown in the right panel of Figure 5. Here we select $\tau_{c}=5 \mathrm{kyr}$ and $r=2 \mathrm{pc}$, representing a young, energetic and large PWN. While the predicted X-ray flux is now correct, the slope of the synchrotron curve in the NuSTAR band is not. Manipulating the model does not produce an SED curve that correctly matches the detected emission of G359.97-0.038.

Based on the broadband morphological and spectral characteristics of G359.97-0.038, we can conclude that the filament is not a PWN.

\subsection{G359.97-0.038 as a Magnetic Flux Tube}

Another possible origin of G359.97-0.038 that can be discarded is a magnetic flux tube that traps high energy electrons (Yusef-Zadeh et al. 1984; Tsuboi et al. 1986; Zhang et al. 2014). The magnetic field within a flux tube is locally enhanced by roughly one order of magnitude compared to the large-scale GC magnetic field $(\approx 10 \mu \mathrm{G})$ (e.g., Tsuboi et al. 1985). Relativistic electrons that become trapped in the tubes interact with the enhanced magnetic field and radiate via synchrotron emission. Electrons with energies of $10-100 \mathrm{TeV}$ are required to generate $\mathrm{X}$-ray synchrotron emission up to a few tens of $\mathrm{keV}$, assuming 

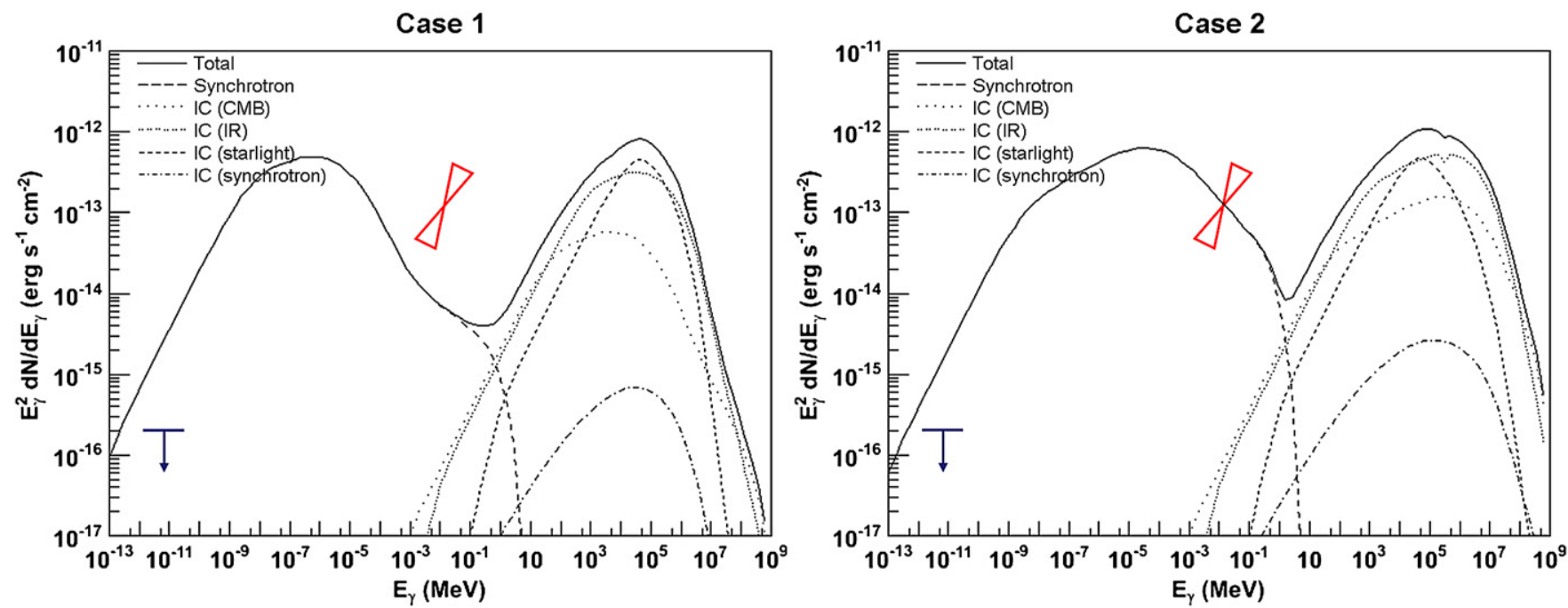

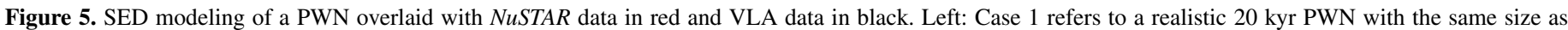
G359.97-0.038. Right: Case 2 depicts a young, large PWN with an energetic pulsar that has approximately the same X-ray flux as G359.97-0.038.

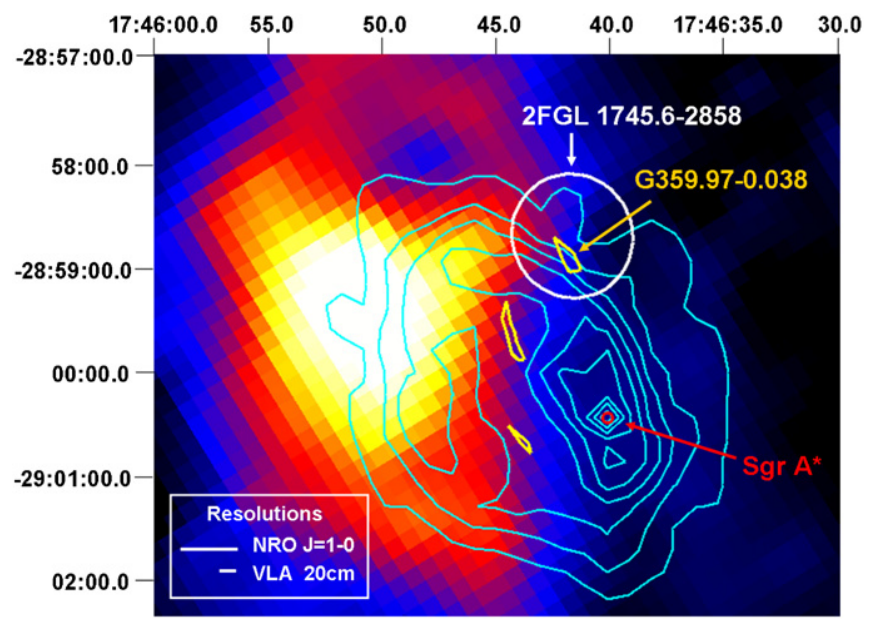

Figure 6. Image of CS $J=1-0$ emission obtained with the NRO $45 \mathrm{~m}$ telescope, which highlights the location of the $50 \mathrm{~km} \mathrm{~s}^{-1}$ cloud (M-02-0.07). Sgr A East contours are shown in cyan, obtained from a $20 \mathrm{~cm}$ VLA map. G359.97-0.038 as well as two other Chandra X-ray filaments are shown in yellow. The large white circle indicates the uncertainty in the position of the Fermi source 2FGL $J 1745.6-2858$. FWHM resolutions are indicated in the lower right corner for reference.

a local magnetic field of $\sim 100 \mu \mathrm{G}$. GeV electrons, in contrast, are required to generate synchrotron emission in the radio band.

Sgr A-E is a known magnetic flux tube bright in the radio and X-ray bands (Zhang et al. 2014; Morris et al. 2014). Zhang et al. (2014) argue that Sgr A-E is likely illuminated by $\sim 10-100 \mathrm{TeV}$ electrons that emanate from the nearby $20 \mathrm{~km} \mathrm{~s}^{-1}$ MC. Local cosmic-ray protons interact with the MC and produce secondary $\mathrm{TeV}$ electrons, which can diffuse out and become trapped by the adjacent flux tube. G359.97-0.038 is adjacent to several MCs such as the $50 \mathrm{~km} \mathrm{~s}^{-1}$ cloud M-0.02-0.07 shown in Figure 6, which can be sources of energetic electrons for the filament.

As with the PWN theory, however, a magnetic flux tube origin can be eliminated as a possible explanation for G359.97-0.038 through broadband spectral and spatial analysis.

The morphology of G359.97-0.038 does not resemble that of a magnetic flux tube. The radio emission of Sgr A-E is more extended than that of the X-ray emission, consistent with electrons subject to synchrotron cooling losses. G359.97-0.038 does not have such a morphology, as shown in Figure 2. If the feature is indeed the radio counterpart of G359.97-0.038 then the more compressed radio emission is difficult to explain by synchrotron emission unless there is a very different spatial distribution of the $\mathrm{GeV}$ and $\mathrm{TeV}$ electrons, which are responsible for the radio and X-ray emission, respectively. Additionally, Sgr A-E has regions of brightened radio and X-ray emission at the location where several of the flux tubes intersect. In contrast, the morphology of G359.97-0.038 has its bright point-like emission positioned on a single filamentary structure.

Additionally, the spectral evidence does not support G359.970.038 as a magnetic flux tube. There are no existing models predicting the spectra of electrons escaped from particular MCs including the $50 \mathrm{~km} \mathrm{~s}^{-1}$ cloud. Although we have few constraints on the range of the power-law index of the escaped electrons, we note that Sgr A-E has a photon index of $\Gamma \sim$ $2.3 \pm 0.1$ (Zhang et al. 2014), which is significantly softer than G359.97-0.038.

While the magnetic flux tube scenario cannot be definitively ruled out as an explanation for G359.97-0.038, its morphological and spectral characteristics make this scenario highly unlikely.

\subsection{G359.97-0.038 as SNR-MC Interaction}

A plausible explanation is that G359.97-0.038 results from the interaction between the SN shell of Sgr A East and nearby MCs. It is known that the far side of Sgr A East is pushing into the $50 \mathrm{~km} \mathrm{~s}^{-1} \mathrm{MC} \mathrm{M-0.02-0.07} \mathrm{(Tsuboi} \mathrm{et} \mathrm{al.} \mathrm{2006,} \mathrm{2009;}$ Herrnstein \& Ho 2005). We examine the likely possibility that G359.97-0.038 is also the result of an SNR-MC interaction site.

Bykov et al. (2000) developed a theory that describes the broadband spectrum of an SNR-MC interaction. As a shock wave from the SN propagates into the $\mathrm{MC}$, first- and secondorder Fermi accelerations produce non-thermal electrons that radiate via synchrotron, bremsstrahlung, and IC scattering, though only the latter two emission mechanisms are important at the energies observed by Chandra and NuSTAR. Bykov and coauthors analyze three possible mechanisms that produce nonthermal emission: a thermal population of electrons accelerated via radiative shocks; Galactic cosmic-ray protons accelerated 
via radiative shock; and a forward shock propagating into a dense MC clump. The resultant SED models and detected emission from IC 443 are seen in Figures 1-3 of Bykov et al. (2000). All three are characterized by synchrotron emission in the radio band with non-thermal bremsstrahlung emission from $\sim \mathrm{keV}$ to $\mathrm{TeV}$ produced by neutral pion decay. The X-ray spectrum of IC 443 has a photon index of $\Gamma \sim 1.0$, and its SED curve begins to rise sharply at X-ray energies. NuSTAR and Chandra are well-suited to capture this range. At higher energies, the predicted flux peaks around $0.1-2.0 \mathrm{GeV}$ with a $\mathrm{GeV}$ photon index of $\Gamma \sim 2$, after which the $\gamma$-ray emission drops sharply and becomes negligible above $\sim 1 \mathrm{TeV}$. The models are supported by observational data from known SNR-MC interactions such as IC 443 (Bykov et al. 2000) and W82 (Fujita et al. 2009).

The exact model spectra reported by Bykov et al. (2000) depend on parameters such as cloud density and shock velocity that are specific to IC 443. IC 443 is middle-aged SNR estimated to be approximately 10-30 kyr old (e.g., Petre et al. 1988; Lee et al. 2008), comparable to the $\sim 10 \mathrm{kyr}$ old Sgr A East (Maeda et al. 2002; Zhao et al. 2013). It is informative to compare the broadband emission spanning the radio, X-ray, and $\gamma$-ray energy bands from a known SNR-MC object to that observed by G359.97-0.038 in order to see if there are any parallel characteristics.

Morphological features detected in IC 443 are similar to those in G359.97-0.038. The large SNR has several bright point-like X-ray objects that are located near the region where the SN shock front interacts with the dense MC (Bocchino \& Bykov 2003), and recent XMM-Newton observations revealed a narrow "bridge" extending from one of those objects, XMM J061804.3+222732 (Bocchino et al. 2008). This resembles G359.97-0.038: both are comprised of a bright compact feature with an extended filamentary structure in the X-ray band.

We can also compare the spectral parameters of G359.970.038 to those of IC 443 . While the IC 443 models invoking accelerated thermal electrons and cosmic-ray protons match well with the NuSTAR and Chandra data from G359.97-0.038, the predicted radio flux is over three orders of magnitude higher than what is observed in G359.97-0.038. As mentioned in Section 5.2, the detected radio of G359.97-0.038 emission represents an upper limit, and the discrepancy between the IC 443 models and the radio band emission from G359.97-0.038 may be even greater. We therefore turn to the model that represents the interaction between the radiative shock front and dense MC clumps shown in Figure 1 reported by Bykov et al. (2000).

Comparison of the X-ray emission shows that the NuSTAR data is reasonably similar to what is predicted by the model. The $N U S T A R$ photon index of $\Gamma=1.3 \pm 0.3$ is consistent with the predicted IC 443 photon index of $\Gamma \sim 1.0$ (Bykov et al. 2000). The X-ray and radio luminosities are also consistent. Thus the $\mathrm{X}$-ray and radio data of G359.97-0.038 correlate well with the IC 443 model.

Further support for this model might be obtained if there were associated $\gamma$-ray emission. The Fermi observatory discovered a source (2FGL J1745.6-2858) coincident with G359.97-0.038 (Nolan et al. 2012). This source was originally reported as 1FGL J1745.6-2900c in the first-year Fermi Large Area Telescope catalog with a location whose $95 \%$ position uncertainty did not overlap G359.97-0.038 (Abdo et al. 2010). Rather, the source was coincident with the GC and was tentatively associated with the large PWN near Sgr A*, G359.95-0.04. An updated background model later reported by Nolan et al. (2012) presented a new centroid position of the $\gamma$-ray point source coincident with G359.97-0.038. The 95\% positional uncertainty is shown in Figure 6 . The location is confirmed with an independent analysis by Yusef-Zadeh et al. (2013). The same source is also identified in the first Fermi High-energy catalog (1 FHL; The Fermi-LAT Collaboration 2013), which analyzes $E>10 \mathrm{GeV}$ emission.

The $\gamma$-ray spectrum of 2FGL J1745.6-2858 is well fit with either a log-parabolic model (Nolan et al. 2012) or a broken power-law model (Yusef-Zadeh et al. 2013). Each produces a power-law index of $\Gamma \sim 2.0, v F_{v} \sim 80 \mathrm{ev} \mathrm{cm}^{-2} \mathrm{~s}^{-1}$, and a peak energy at $\sim 2-3 \mathrm{GeV}$. The SED model presented by Bykov et al. (2000) predicts a peak at around $\sim 1 \mathrm{GeV}$, after which the bremsstrahlung emission sharply decreases.

If the $\gamma$-ray emission is associated with G359.97-0.038, then it follows this prediction in spectral shape by a factor of $\sim 3$. However, several model parameters influence this $\gamma$-ray behavior, notable among them the maximum energy of the accelerated electrons, as seen in Figure 3 of Bykov et al. (2000). Additionally, G359.97-0.038 is close to the GC, which has high density IR and UV photons that can add IC emission to the bremsstrahlung spectrum predicted by the SNR-MC interaction, increasing the observed GeV flux.

The complexity of the GC does not allow for firm identification of which MC is responsible for the emission of G359.970.038 . The presence of an $\mathrm{OH}$ maser is a strong indicator of an SNR-MC shock interaction (Pihlström \& Sjouwerman 2006) and as such can trace the approximate locations of the interaction sites. A collection of bright $\mathrm{OH} 1720 \mathrm{MHz}$ masers are located near the Eastern boundary of Sgr A East delimiting where the SNR is expanding into M-0.02-0.07. G359.97-0.038 is positioned toward the NW of the Sgr A East radio shell. Multiple $\mathrm{OH}$ masers are also detected in this region, one located $\sim 15^{\prime \prime} \mathrm{SE}$ from the bright point-like object in G359.97-0.038, at R.A. $=17^{\mathrm{h}} 45^{\mathrm{m}} 42^{\mathrm{s}}$, decl. $=-28^{\circ} 59^{\prime} 10^{\prime \prime}(\mathrm{J} 2000)$ with a velocity of $+34 \mathrm{~km} \mathrm{~s}^{-1}$ (Yusef-Zadeh et al. 2001; Sjouwerman \& Pihlström 2008). Sjouwerman \& Pihlström (2008) claim this is likely evidence of Sgr A East interacting with either M-0.020.07 or the $20 \mathrm{~km} \mathrm{~s}^{-1} \mathrm{MC} \mathrm{M}-0.13-0.08$, though other higher velocity clouds (e.g., Serabyn et al. 1986) are also possible.

While further analysis is required to determine which MC is responsible for G359.97-0.038, the broadband spectral and spatial features of the emission support the claim that G359.970.038 is the result of the SNR Sgr A East interacting with a nearby $\mathrm{MC}$.

\section{SUMMARY}

NuSTAR observations extend the previous X-ray detection of the NTF G359.97-0.038 from $\sim 8 \mathrm{keV}$ to $\sim 40 \mathrm{keV}$. This is the second NTF detected in the hard X-ray band by NuSTAR in its survey of the GC region. The best-fit non-thermal spectrum is characterized by a photon index of $\Gamma=1.3_{-0.2}^{+0.3}$ and a luminosity of $2.6 \times 10^{33} \mathrm{erg} \mathrm{s}^{-1}$ from $20-40 \mathrm{keV}$. Previously it was suggested that this NTF was a PWN. However, the X-ray and radio morphology, along with the broadband spectral energy distribution, are not typical of a PWN. Analyses of deep Chandra observations also show no evidence of an X-ray synchrotron cooling tail. The radio and X-ray morphology are also shown to be inconsistent with a magnetic flux tube origin, despite the proximity of G359.97-0.038 to an MC that could serve as a source of energetic electrons. The most likely explanation for G359.97-0.038 is an interaction of the SNR Sgr A-E with a nearby $50 \mathrm{~km} \mathrm{~s}^{-1} \mathrm{MC}$. The interaction of this middle-aged 
SNR with the MC is supported by the broadband spectral energy distribution, radio and X-ray morphology, and presence of masing action indicative of shock interaction. This NTF closely resembles a region of IC 443, where a similar interaction is occurring. While X-ray emitting NTFs are generally believed to be PWNe, neither of the NTFs so far detected by NuSTAR, G359.97-0.038 reported here and Sgr A-E reported in Zhang et al. (2014), have their origin in PWNe.

This work was supported under NASA contract No. NNG08FD60C, and made use of data from the NuSTAR mission, a project led by the California Institute of Technology, managed by the Jet Propulsion Laboratory, and funded by the National Aeronautics and Space Administration. F.E.B. acknowledges support from CONICYT-Chile (Basal-CATA PFB-06/2007, FONDECYT 1141218, "EMBIGGEN" Anillo ACT1101), and Project IC120009 "Millennium Institute of Astrophysics (MAS)" funded by the Iniciativa Científica Milenio del Ministerio de Economía, Fomento y Turismo. We thank the NuSTAR Operations, Software, and Calibration teams for support with the execution and analysis of these observations. This research has made use of the NuSTAR Data Analysis Software (NuSTARDAS) jointly developed by the ASI Science Data Center (ASDC, Italy) and the California Institute of Technology (USA).

\section{REFERENCES}

Abdo, A. A., Ackermann, M., Ajello, M., et al. 2010, ApJS, 188, 405

An, H., Madsen, K. K., Reynolds, S. P., et al. 2014, ApJ, 793, 90

Baganoff, F. K., Maeda, Y., Morris, M., et al. 2003, ApJ, 591, 891

Barrière, N. M., Tomsick, J. A., Baganoff, F. K., et al. 2014, ApJ, 786, 46

Bocchino, F., \& Bykov, A. M. 2003, A\&A, 400, 203

Bocchino, F., Krassilchtchikov, A. M., Kretschmar, P., et al. 2008, AdSpR, 41, 396

Broos, P. S., Townsley, L. K., Feigelson, E. D., et al. 2010, ApJ, 714, 1582

Bykov, A. M., Chevalier, R. A., Ellison, D. C., \& Uvarov, Y. A. 2000, ApJ, 538, 203

Fruscione, A., McDowell, J. C., Allen, G. E., et al. 2006, Proc. SPIE, $6270,62701 \mathrm{~V}$

Fujita, Y., Ohira, Y., Tanaka, S. J., \& Takahara, F. 2009, ApJL, 707, L179

Gaensler, B. M., \& Slane, P. O. 2006, ARA\&A, 44, 17

Gaensler, B. M., van der Swaluw, E., Camilo, F., et al. 2004, ApJ, 616,383

Goldwurm, A. 2011, in ASP Conf. Ser. 439, The Galactic Center: A Window to the Nuclear Environment of Disk Galaxies, ed. M. R. Morris, Q. D. Wang, \& F. Yuan (San Francisco, CA: ASP), 391

Harrison, F. A., Craig, W. W., Christensen, F. E., et al. 2013, ApJ, 770, 103
Herrnstein, R. M., \& Ho, P. T. P. 2005, ApJ, 620, 287

Inoue, M., Takahashi, T., Tabara, H., Kato, T., \& Tsuboi, M. 1984, PASJ, 36,633

Johnson, S. P., Dong, H., \& Wang, Q. D. 2009, MNRAS, 399, 1429

Kargaltsev, O., \& Pavlov, G. G. 2008, in AIP Conf. Ser. 983, 40 Years of Pulsars: Millisecond Pulsars, Magnetars and More, ed. C. Bassa, Z. Wang, A. Cumming, \& V. M. Kaspi (Melville, NY: AIP), 171

Krivonos, R. A., Tomsick, J. A., Bauer, F. E., et al. 2014, ApJ, 781, 107

Lee, J.-J., Koo, B.-C., Yun, M. S., et al. 2008, AJ, 135, 796

Lu, F. J., Wang, Q. D., \& Lang, C. C. 2003, AJ, 126, 319

Lu, F. J., Yuan, T. T., \& Lou, Y.-Q. 2008, ApJ, 673, 915

Maeda, Y., Baganoff, F. K., Feigelson, E. D., et al. 2002, ApJ, 570, 671

Mori, K., Gotthelf, E. V., Zhang, S., et al. 2013, ApJL, 770, L23

Morris, M. R., Zhao, J.-H., \& Goss, W. M. 2014, in IAU Symp. 303, The Galactic Center: Feeding and Feedback in a Normal Galactic Nucleus, ed. L. O. Sjouwerman, C. C. Lang, \& J. Ott (Cambridge: Cambridge Univ. Press), 369

Muno, M. P., Baganoff, F. K., Brandt, W. N., Morris, M. R., \& Starck, J.-L. 2008, ApJ, 673, 251

Nolan, P. L., Abdo, A. A., Ackermann, M., et al. 2012, ApJS, 199, 31

Nynka, M., Hailey, C. J., Mori, K., et al. 2013, ApJL, 778, L31

Nynka, M., Hailey, C. J., Reynolds, S. P., et al. 2014, ApJ, 789, 72

Perez, K., Hailey, C. J., Bauer, F. E., et al. 2015, Natur, in press

Petre, R., Szymkowiak, A. E., Seward, F. D., \& Willingale, R. 1988, ApJ, 335,215

Pihlström, Y. M., \& Sjouwerman, L. O. 2006, JPhCS, 54, 77

Reich, W., Sofue, Y., \& Matsuo, H. 2000, PASJ, 52, 355

Reynolds, S. P. 2009, ApJ, 703, 662

Sakano, M., Warwick, R. S., Decourchelle, A., \& Predehl, P. 2004, MNRAS, 350,129

Serabyn, E., Guesten, R., Walmsley, J. E., Wink, J. E., \& Zylka, R. 1986, A\&A, 169,85

Sjouwerman, L. O., \& Pihlström, Y. M. 2008, ApJ, 681, 1287

The Fermi-LAT Collaboration. 2013, arXiv:1306.6772

Tsuboi, M., Inoue, M., Handa, T., Tabara, H., \& Kato, T. 1985, PASJ, 37, 359

Tsuboi, M., Inoue, M., Handa, T., et al. 1986, AJ, 92, 818

Tsuboi, M., Miyazaki, A., \& Okumura, S. K. 2009, PASJ, 61, 29

Tsuboi, M., Okumura, S. K., \& Miyazaki, A. 2006, JPhCS, 54, 16

Verner, D. A., Ferland, G. J., Korista, K. T., \& Yakovlev, D. G. 1996, ApJ, 465,487

Wang, Q. D., Lu, F. J., \& Gotthelf, E. V. 2006, MNRAS, 367, 937

Wik, D. R., Hornstrup, A., Molendi, S., et al. 2014, ApJ, 792, 48

Wilms, J., Allen, A., \& McCray, R. 2000, ApJ, 542, 914

Yusef-Zadeh, F., Hewitt, J. W., Wardle, M., et al. 2013, ApJ, 762, 33

Yusef-Zadeh, F., Morris, M., \& Chance, D. 1984, Natur, 310, 557

Yusef-Zadeh, F., Stolovy, S. R., Burton, M., Wardle, M., \& Ashley, M. C. B. 2001, ApJ, 560, 749

Yusef-Zadeh, F., Wardle, M., Muno, M., Law, C., \& Pound, M. 2005, AdSpR, 35,1074

Zhang, L., Chen, S. B., \& Fang, J. 2008, ApJ, 676, 1210

Zhang, S., Hailey, C. J., Baganoff, F. K., et al. 2014, ApJ, 784, 6

Zhao, J.-H., Morris, M. R., \& Goss, W. M. 2013, ApJ, 777, 146

Zhao, J.-H., Morris, M. R., Goss, W. M., \& An, T. 2009, ApJ, 699, 186 